PDES, SUBMANIFOLDS AND

AFFINE DIFFERENTIAL GEOMETRY

BANACH CENTER PUBLICATIONS, VOLUME 57

INSTITUTE OF MATHEMATICS

POLISH ACADEMY OF SCIENCES

WARSZAWA 2002

\title{
SURFACES WITH PARALLEL SECOND FUNDAMENTAL FORM IN BIANCHI-CARTAN-VRANCEANU SPACES
}

\author{
MOHAMED BELKHELFA \\ Departement Wiskunde, Katholieke Universiteit Leuven \\ Celestijnenlaan 200B, B-3001 Heverlee, Belgium \\ E-mail: mohamed.belkhelfa@wis.kuleuven.ac.be \\ FRANKI DILLEN \\ Departement Wiskunde, Katholieke Universiteit Leuven \\ Celestijnenlaan 200B, B-3001 Heverlee, Belgium \\ E-mail: franki.dillen@wis.kuleuven.ac.be \\ JUN-ICHI INOGUCHI \\ Departement of Applied Mathematics, Fukuoka University \\ Fukuoka 814-0180, Japan \\ E-mail: inoguchi@bach.sm.fukuoka-u.ac.jp
}

\begin{abstract}
We give a complete classification of surfaces with parallel second fundamental form in 3-dimensional Bianchi-Cartan-Vranceanu spaces.

1. Introduction. Submanifolds with parallel second fundamental form in Euclidean space play an important role in differential geometry. In fact such submanifolds are symmetric $R$-spaces and imbedded as locally extrinsically symmetric submanifolds [26], [43].

When the ambient space is not symmetric, the condition "parallel second fundamental form" is a strong restriction. In fact even if the ambient space is homogeneous, surfaces with parallel second fundamental form are very few. For instance, we have proved that the only surfaces with parallel second fundamental form in the 3-dimensional Heisenberg group or the special linear group $\mathrm{SL}_{2} \mathbb{R}$ are Hopf cylinders [4], [5].
\end{abstract}

2000 Mathematics Subject Classification: Primary 53A99; Secondary 53C42, 53D15.

Key words and phrases: Bianchi-Cartan-Vranceanu metrics, contact space forms, surfaces with parallel second fundamental form.

The first named author is supported by the Fund for Scientific Research - Flanders (Belgium) (F.W.O.) through project G. 0366. 98.

The third named author is partially supported by Grand-in-Aid for Encouragement of Young Scientists, No. 12740051, Japan Society for Promotion of Science.

The paper is in final form and no version of it will be published elsewhere. 
Both the Heisenberg group and $\mathrm{SL}_{2} \mathbb{R}$ are contact space forms, i.e., complete and connected Sasaki manifolds of constant holomorphic sectional curvature. More precisely every simply connected contact space form of holomorphic sectional curvature $c=-3$ [resp. $c<-3]$ is isomorphic to the Heisenberg group [resp. the universal covering of $\left.\mathrm{SL}_{2} \mathbb{R}\right]$.

Every 3-dimensional simply connected contact space form with holomorphic sectional curvature greater than -3 is isomorphic to unit 3 -sphere $S^{3}$ with canonical Sasaki structure and its $D$-homothetic deformation. As Riemannian manifolds, such contact space forms are represented as special unitary group $\mathrm{SU}(2)$ with specified left invariant metric.

The contact space forms have been studied in some contexts. For instance, Euclidean 3 -space and simply connected contact 3-space forms are included in the so called BianchiCartan-Vranceanu family of 3-dimensional homogeneous Riemannian manifolds ([11], $[12],[21],[18],[19],[28],[52])$, the classification of 3-dimensional naturally reductive spaces [50] and the classification file of Thurston's 3-dimensional model geometries [48].

It seems to be interesting to give a complete classification of surfaces with parallel second fundamental form in 3-dimensional contact space forms. For these reasons, we shall study surfaces with parallel second fundamental form in homogeneous Riemannian 3-manifolds with 4-dimensional isometry group.

This paper has two purposes. Firstly, we shall exhibit explicit matrix group models of 3-dimensional contact space forms. Furthermore we shall give explicit models of 3-dimensional homogeneous spaces with Bianchi-Cartan-Vranceanu metric and their associated almost contact structures.

In recent years, geometry of surfaces in a 3-dimensional homogeneous space with Bianchi-Cartan-Vranceanu metric has been studied extensively.

For example Caddeo, Piu and Ratto [18], [19] studied surfaces of revolution with constant mean or Gaussian curvature in the Heisenberg group. Tomter also studied surfaces of revolution with constant mean curvature in the Heisenberg group [49].

Kokubu [34] studied rotational surfaces and conoids of constant mean curvature in $\mathrm{SL}_{2} \mathbb{R}$. Kikuchi [32] studied $S^{1}$-equivariant constant mean curvature tori in elliptic contact space form.

In [29], a representation formula for minimal surfaces in Heisenberg group is given.

The second purpose of this paper is to generalize the classification theorem for parallel surfaces in the Heisenberg group [4] and the special linear group $\mathrm{SL}_{2} \mathbb{R}$ [5] to simply connected 3-dimensional homogeneous Riemannian 3-manifolds with Bianchi-CartanVranceanu metrics.

2. Contact manifolds. Let $M$ be a $(2 n+1)$-manifold. A one form $\eta$ is called a contact form on $M$ if $(d \eta)^{n} \wedge \eta \neq 0$. A manifold $M$ together with a contact form is called a contact manifold. The distribution $D$ defined by

$$
D=\{X \in T M \mid \eta(X)=0\}
$$

is called the contact structure or contact distribution determined by $\eta$. 
On a contact manifold $(M, \eta)$, there exists a unique vector field $\xi$ such that

$$
\eta(\xi)=1, \quad d \eta(\xi, \cdot)=0 .
$$

Namely $\xi$ is transversal to the contact structure $D$. This vector field $\xi$ is called the Reeb vector field or characteristic vector field of $(M, \eta)$.

Moreover there exists an endomorphism field $\varphi$ and a Riemannian metric $g$ on $M$ such that

$$
\begin{gathered}
\varphi^{2}=-I+\eta \otimes \xi, \eta(\xi)=1, \\
g(\varphi X, \varphi Y)=g(X, Y)-\eta(X) \eta(Y), \quad g(\xi, \cdot)=\eta, \\
d \eta(X, Y)=2 g(X, \varphi Y)
\end{gathered}
$$

for all vector fields $X, Y$ on $M$. The pair $(\varphi, g)$ (or quartet $(\eta, \xi, \varphi, g)$ ) is called the associated almost contact structure of $(M, \eta)$. More generally an odd dimensional manifold $M^{2 n+1}$ is said to be an almost contact manifold if there exists a triplet $(\eta, \xi, \varphi)$ satisfying (2.1). Note that the existence of an almost contact structure is equivalent to the reducibility of the structure group of the tangent bundle $T M$ of $M$ to $\mathrm{U}(n) \times\{1\}$. On an almost contact manifold $(M, \eta ; \xi, \varphi)$, there exists a Riemannian metric $g$ satisfying $(2.2)$. Such a metric $g$ is called a compatible metric of $M$.

Next, on the direct product $M \times \mathbb{R}(t)$ of an almost contact manifold $M$ and real line $\mathbb{R}(t)$, we can extend naturally the endomorphism field $\varphi$ to an almost complex structure $J$ on $M \times \mathbb{R}(t)$ :

$$
J\left(X, f \frac{\partial}{\partial t}\right)=\left(\varphi X-f \xi, \eta(X) \frac{\partial}{\partial t}\right), X \in \mathfrak{X}(M), f \in C^{\infty}(M \times \mathbb{R}) .
$$

If the almost complex structure $J$ on $M \times \mathbb{R}$ is integrable then $(M, \eta)$ is said to be normal. The normality is equivalent to the vanishing of the Sasaki torsion $\mathcal{N}_{1}$ :

$$
\mathcal{N}_{1}(X, Y)=[\varphi, \varphi](X, Y)+d \eta(X, Y) .
$$

Here $[\varphi, \varphi]$ is the Nijenhuis torsion of $\varphi$ :

$$
[\varphi, \varphi](X, Y)=\varphi^{2}[X, Y]+[\varphi X, \varphi Y]-\varphi[\varphi X, Y]-\varphi[X, \varphi Y], \quad X, Y \in \mathfrak{X}(M) .
$$

A normal contact manifold $(M, \eta ; \xi, \varphi, g)$ is called a Sasaki manifold. Note that the notion of Sasaki manifold is equivalent to that of normal strongly pseudo convex $C R$ manifold [45].

Proposition 2.1. Let $(M, \eta, \xi, \varphi, g)$ be an almost contact manifold. Then $M$ is a contact manifold with associated metric (2.3) and $\xi$ is a Killing vector field if and only if

$$
\nabla_{X} \xi=-\varphi X, \quad X \in \mathfrak{X}(M) .
$$

An almost contact manifold $M$ is a Sasaki manifold if and only if

$$
\left(\nabla_{X} \varphi\right) Y=g(X, Y) \xi-\eta(Y) X, \quad X, Y \in \mathfrak{X}(M) .
$$

Note that on a Sasaki manifold, $\xi$ is Killing.

To close this section, we shall prepare some fundamental facts on Hopf cylinders in regular almost contact 3 -manifolds. 
Let $(M, \eta ; \xi, \varphi, g)$ be an almost contact manifold. Then $M$ is said to be regular if $\xi$ generates a one-parameter group $K$ of isometries on $M^{2 n+1}$, such that the action of $K$ on $M^{2 n+1}$ is simply transitive and structure tensors $\varphi$ and $\eta$ are invariant under $K$-action. If $M$ is contact then $\varphi$ and $\eta$ are automatically $K$-invariant, i.e, $£_{\xi} \varphi=0$ and $£_{\xi} \eta=0$. The Killing vector field $\xi$ induces a regular one-dimensional Riemannian foliation on $M$. We denote by $\tilde{M}:=M / \xi$ the orbit space (the space of all leaves) of a regular almost contact manifold $M$ under the $K$-action.

The almost contact structure on the regular almost contact manifold induces an almost Hermitian structure on the orbit space $\tilde{M}$. Further the natural projection $\pi: M \rightarrow \tilde{M}$ is a Riemannian submersion. It is easy to see that $M$ is contact if and only if $\tilde{M}$ is almost Kähler (symplectic). In addition, $M$ is Sasaki if and only if $\tilde{M}$ is Kähler ( $c f$. Hatakeyama [27], Ogiue [38]).

On the other hand, a complete and regular almost contact manifold $M$ satisfies $d \eta=0$ if and only if $M$ is isomorphic to the direct product $\tilde{M} \times \mathbb{R}$ or $\tilde{M} \times S^{1}$, see Lemma 2 in [14]. In this case $\xi$ is parallel.

In 1958, W. M. Boothby and H. C. Wang [16] proved that if $M$ is a compact regular contact manifold, then the natural projection $\pi: M \rightarrow \tilde{M}$ defines a principal circle bundle over a symplectic manifold $\tilde{M}$ and the symplectic form $\Phi$ of $\tilde{M}$ determines an integral cocycle. Furthermore the contact form $\eta$ gives a connection form of this circle bundle and satisfies $\pi^{*} \Phi=d \eta$. The fibering $\pi: M \rightarrow \tilde{M}$ is called the Boothby-Wang fibering of a regular compact contact manifold $M$.

In particular for a compact regular Sasaki manifold, the orbit space $\tilde{M}$ is a Hodge manifold, i.e., a compact Kähler manifold whose Kähler form $\Phi$ is of bidegree $(1,1)$ and determines an integral cocycle. (A. Morimoto [36]. See also [38].) Hence $M$ is a circle bundle over a projective algebraic manifold $\tilde{M}$.

The unit sphere $S^{2 n+1}$ is a typical example of regular compact Sasaki manifolds. For $S^{2 n+1}$, the Boothby-Wang fibering coincides with the Hopf fibering $S^{2 n+1} \rightarrow \mathbb{C} P^{n}$.

Now we shall restrict our attention to 3 -dimensional regular almost contact manifold $M^{3}$. In the rest of this section, we assume that the regular almost contact manifold $M$ is contact, the direct product $\tilde{M} \times \mathbb{R}$ or $\tilde{M} \times S^{1}$. Let $\tilde{\gamma}$ be a curve parameterized by arc length in $\tilde{M}$ with curvature $\tilde{\kappa}$. Taking the inverse image $S:=\pi^{-1}(\tilde{\gamma})$ of $\tilde{\gamma}$ in $M^{3}$, then it is easy to see that $S$ is a flat surface in $M^{3}$.

We shall call this surface $S$ the Hopf cylinder (or Boothby-Wang cylinder) over $\tilde{\gamma}$. The mean curvature $H$ of $M$ is $\tilde{\kappa} / 2$. In particular if $\tilde{\gamma}$ is closed, then $S$ is called the Hopf torus over $\tilde{\gamma}$.

U. Pinkall [40] used this construction in his study on flat tori in 3-sphere $S^{3}$. In $S^{3}$ case if we take a geodesic $\tilde{\gamma}$ in $S^{2}(1 / 2)$, then the Hopf torus over $\tilde{\gamma}$ is the Clifford minimal torus.

For more details on contact manifolds, we refer to Blair's lecture notes [13].

3. Contact space forms. Let $(M, \eta ; \xi, \varphi, g)$ be an almost contact manifold. A tangent plane at a point of $M$ is said to be a holomorphic plane if it is invariant under $\varphi$. The sectional curvature of a holomorphic plane is called holomorphic sectional curvature. 
If the sectional curvature function of $M$ is constant on all holomorphic planes in $T M$, then $M$ is said to be of constant holomorphic sectional curvature.

Complete and connected Sasaki manifolds of constant holomorphic sectional curvature are called contact space forms or Sasakian space forms. Let $M^{2 n+1}$ be a contact space form of constant holomorphic sectional curvature $c$. Then $M$ is said to be an elliptic [resp. parabolic or hyperbolic] contact space form if $c>-3$ [resp. $c=-3$ or $c<-3$.]

S. Tanno classified simply connected contact space forms. Further he gave model spaces for simply connected contact space forms.

Proposition 3.1 ([46]). Let $\mathcal{M}^{2 n+1}(c)$ be a simply connected contact space form. Then $\mathcal{M}^{2 n+1}$ is isomorphic to Heisenberg group $N^{2 n+1}$ with canonical Sasaki structure if $c=-3$, and $\mathcal{M}^{2 n+1}(c)$ is isomorphic to $\mathbb{C} H^{n} \times \mathbb{R}$ if $c<-3$. Here $\mathbb{C} H^{n}$ is the complex hyperbolic $n$-space.

We shall recall Tanno's explicit construction of simply connected elliptic contact space form $\mathcal{M}^{2 n+1}(c), c>-3$. See also V. N. Berestovskiur and D. E. Vol'per [9] and T. Sakai [41].

Let us denote by $\left(\eta_{1}, \xi_{1}, \varphi_{1}, g_{1}\right)$ the canonical contact structure of unit sphere $S^{2 n+1}$. For any positive number $\alpha$, the deformation:

$$
\eta_{\alpha}:=\alpha \eta_{1}, \quad \xi_{\alpha}:=\xi_{1} / \alpha, \quad \varphi_{\alpha}=\varphi_{1}, g_{\alpha}:=\alpha g_{1}+\alpha(\alpha-1) \eta_{1} \otimes \eta_{1}
$$

gives another contact structure on $S^{2 n+1}$. This deformation is called the D-homothetic deformation of $S^{2 n+1}$.

One can easily check that the $D$-homothetic deformation of $S^{2 n+1}$ is a Sasaki manifold of constant holomorphic sectional curvature $c=4 / \alpha-3$. Tanno classified the simply connected elliptic contact space forms. (See also [13], pp. 99-100.)

Proposition 3.2 ([46]). Every simply connected $(2 n+1)$-dimensional elliptic contact space form $\mathcal{M}^{2 n+1}(c)$ is isomorphic to a D-homothetic deformation of the unit sphere $S^{2 n+1}$ with $c=4 / \alpha-3$.

Note that a $D$-homothetic deformation of $S^{2 n+1}$ is a Berger sphere.

Every contact space form $\mathcal{M}^{2 n+1}(c)$ is realised as a real hypersurface in a complex space form $\mathcal{M}^{2 n+2}(c-1)$ with constant holomorphic sectional curvature $c-1$. See J. Berndt [6], [7] and N. Ejiri [25]:

- $c>1: \mathcal{M}^{2 n+1}(c)$ is the geodesic sphere of radius $r=\tan ^{-1}(\sqrt{c-1} / 2)$ in complex projective $(n+1)$-space $\mathbb{C} P^{n+1}$ of constant holomorphic sectional curvature $c-1$,

- $c=1: \mathcal{M}^{2 n+1}(c)$ is the unit sphere in complex Euclidean space $\mathbb{C}^{n+1}$,

- $-3<c<1: \mathcal{M}^{2 n+1}(c)$ is the geodesic sphere of radius $r=\tanh ^{-1}(\sqrt{1-c} / 2)$ in complex hyperbolic $(n+1)$-space $\mathbb{C} H^{n+1}$ of constant holomorphic sectional curvature $c-1$,

- $c=-3: \mathcal{M}^{2 n+1}(c)$ is the horosphere in complex hyperbolic $(n+1)$-space $\mathbb{C} H^{n+1}$ of constant holomorphic sectional curvature -4 ,

- $c<-3: \mathcal{M}^{2 n+1}(c)$ is the universal covering of a tube with radius $r=\operatorname{coth}^{-1}(\sqrt{1-c} / 2)$ around a totally geodesic complex hyperbolic hyperplane in $\mathbb{C} H^{n+1}$ of constant holomorphic sectional curvature $c-1$. 
REMARK 3.3. (1) H. Urakawa [51] studied the spectrum set of $S^{2 n+1}$ with Riemannian metric $g(t)=g_{\alpha} / t^{2(n+1)}, \alpha=t^{2 n+1}, t>0$. Furthermore H. Muto [37] and S. Tanno [47] studied the harmonic stability of $\left(S^{2 n+1}, g(t)\right)$. Namely stability of the identity map with respect to the energy functional. Note that the sectional curvature of $\left(S^{2 n+1}, g(t)\right)$ is positive if $0<t<(4 / 3)^{1 / 3}$ and the deformation $g_{1} \mapsto g(t)$ preserves the volume element of $g_{1}[47]$.

(2) M. Barros [3] studied Willmore surfaces in $\left(S^{3}, g_{\alpha} / \alpha\right), \alpha>0$. The deformation $g_{1} \mapsto g_{\alpha} / \alpha$ is called the canonical variation of $g_{1}[10]$.

(3) É. Cartan proved that every isoparametric hypersurface in $S^{4}$ with three distinct principal curvatures is congruent to a tube of constant radius around the Veronese surface in $S^{4}$ (the image of the first standard imbedding of $\mathbb{R} P^{2}$ in $S^{4}$ ). Such a hypersurface is called the Cartan hypersurface of $S^{4}$. The Cartan hypersurface of $S^{4}$ is extrinsically homogeneous and represented as $\mathrm{SO}(3) / \mathbb{Z}_{2} \times \mathbb{Z}_{2}$.

One can check that the universal Riemannian covering manifold of the Cartan hypersurface is isometric to $\mathcal{M}^{3}(-2)$.

Contact space forms have some remarkable properties. First, contact space forms are naturally reductive homogeneous spaces (so called Sasakian $\varphi$-symmetric spaces) [15]. Next, contact space forms are weakly symmetric spaces (and hence commutative spaces) [8].

In 3-dimensional case, Calvaruso, Perrone and Vanhecke [20] proved that every contact space form is curvature homogeneous. (See Theorem 3.3 in [20].)

Here we recall that F. Tricerri and L. Vanhecke classified 3-dimensional naturally reductive homogeneous Riemannian manifolds. Recall that if $M=G / K$ is a homogeneous manifold, and if $\mathfrak{g}$ and $\mathfrak{k}$ are the Lie algebras of $G$ and $K$ respectively, then $M=G / K$ is said to be reductive if there exists an $\operatorname{Ad}(K)$-invariant subspace $\mathfrak{m}$ of $\mathfrak{g}$ complementary to $\mathfrak{k}$ in $\mathfrak{g}$. The linear subspace $\mathfrak{m}$ is called the Lie subspace of $\mathfrak{g}$. A naturally reductive homogeneous space is a reductive homogeneous manifold $M=(G / K, g)$ furnished with a $G$-invariant Riemannian metric such that for the corresponding inner product $\langle\cdot, \cdot\rangle$ on the Lie subspace $\mathfrak{m}$ we have

$$
\left\langle[X, Y]_{\mathfrak{m}}, Z\right\rangle=\left\langle X,[Y, Z]_{\mathfrak{m}}\right\rangle, \quad X, Y, Z \in \mathfrak{m} .
$$

Here the subscript $\mathfrak{m}$ means the $\mathfrak{m}$-part of vectors.

Proposition 3.4 ([50]). All the simply connected 3-dimensional naturally reductive homogeneous Riemannian manifolds are space forms and the following unimodular Lie groups with special left invariant metric:

The special unitary group $\mathrm{SU}(2)$, Heisenberg group $N^{3}$ and the universal covering group $\widetilde{\mathrm{SL}}_{2} \mathbb{R}$ of $\mathrm{SL}_{2} \mathbb{R}$.

These naturally reductive homogeneous spaces admit a compatible contact structure. More precisely these spaces admit structures of contact space form. In fact simply connected 3-dimensional contact space forms are realized as above the Lie groups with left invariant Sasaki structure ( $c f$. Blair and Vanhecke [14]): 
Corollary 3.5. A simply connected 3-dimensional contact space form is one of the following unimodular Lie groups with left invariant Sasaki structure: the special unitary group $\mathrm{SU}(2)$, the Heisenberg group $N^{3}$ or the universal covering group $\widetilde{\mathrm{SL}_{2}} \mathbb{R}$ of $\mathrm{SL}_{2} \mathbb{R}$.

The only symmetric Sasaki manifold is the unit 3-sphere $S^{3}$ (equivalently $\mathrm{SU}(2)$ with bi-invariant Sasaki structure).

In the following three consecutive sections, we shall exhibit explicit models of 3dimensional contact space forms.

4. Elliptic contact space form. In this section, we shall give an explicit matrix group model of a simply connected elliptic contact space form $\mathcal{M}^{3}(c)$.

As is well known, the unit 3 -sphere $\left(S^{3} ; \eta_{1}, \xi_{1}, \varphi_{1}, g_{1}\right)$ is identified with the special unitary group $G=\mathrm{SU}(2)$ with bi-invariant metric. In this section we shall give a $\mathrm{SU}(2)$ model of $\mathcal{M}^{3}(c)$.

Let us denote the Lie algebra of $G$ by $\mathfrak{g}$. The bi-invariant metric $g_{1}$ of constant curvature 1 on $G$ is induced by the following inner product $\langle\cdot, \cdot\rangle_{1}$ on $\mathfrak{g}$ :

$$
\langle X, Y\rangle_{1}=-\frac{1}{2} \operatorname{tr}(X Y), \quad X, Y \in \mathfrak{g} .
$$

We take a quaternionic basis of $\mathfrak{g}$ :

$$
\mathbf{i}=\left(\begin{array}{cc}
0 & \sqrt{-1} \\
\sqrt{-1} & 0
\end{array}\right), \mathbf{j}=\left(\begin{array}{cc}
0 & -1 \\
1 & 0
\end{array}\right), \quad \mathbf{k}=\left(\begin{array}{cc}
\sqrt{-1} & 0 \\
0 & -\sqrt{-1}
\end{array}\right) .
$$

By using this basis, the group $\mathrm{SU}(2)$ is described as

$$
\mathrm{SU}(2)=\left\{\left(\begin{array}{cc}
x_{0}+\sqrt{-1} x_{3} & -x_{2}+\sqrt{-1} x_{1} \\
x_{2}+\sqrt{-1} x_{1} & x_{0}-\sqrt{-1} x_{3}
\end{array}\right) \mid x_{0}^{2}+x_{1}^{2}+x_{2}^{2}+x_{3}^{2}=1\right\} .
$$

We identify $\mathfrak{g}$ with Euclidean 3 -space $\mathbb{E}^{3}$ via the correspondence

$$
\left(x_{1}, x_{2}, x_{3}\right) \longleftrightarrow x_{1} \mathbf{i}+x_{2} \mathbf{j}+x_{3} \mathbf{k}
$$

Denote the left translated vector fields of $\{\mathbf{i}, \mathbf{j}, \mathbf{k}\}$ by $\left\{E_{1}, E_{2}, E_{3}\right\}$. Then a left invariant Sasaki structure of $G$ is given by

$$
\begin{gathered}
\xi_{1}:=E_{3}, \quad \eta_{1}=g_{1}\left(E_{3}, \cdot\right), \\
\varphi_{1}\left(E_{1}\right)=E_{2}, \quad \varphi_{1}\left(E_{2}\right)=-E_{1}, \quad \varphi_{1}\left(E_{3}\right)=0 .
\end{gathered}
$$

Note that the commutation relations of $\left\{E_{1}, E_{2}, E_{3}\right\}$ are

$$
\left[E_{1}, E_{2}\right]=2 E_{3}, \quad\left[E_{2}, E_{3}\right]=2 E_{1}, \quad\left[E_{3}, E_{1}\right]=2 E_{2} .
$$

The Lie group $G$ acts isometrically on the Lie algebra $\mathfrak{g}$ by the Ad-action.

$$
\text { Ad }: G \times \mathfrak{g} \rightarrow \mathfrak{g} ; \operatorname{Ad}(a) X=a X a^{-1}, \quad a \in G, X \in \mathfrak{g} .
$$

The Ad-orbit of $\mathbf{k} / 2$ is a sphere of radius $1 / 2$ in the Euclidean 3 -space $\mathbb{E}^{3}=\mathfrak{g}$. The Ad-action of $G$ on $S^{2}(1 / 2)$ is isometric and transitive. The isotropy subgroup of $G$ at $\mathbf{k} / 2$ is

$$
\mathrm{U}(1)=\left\{\left(\begin{array}{cc}
e^{\sqrt{-1} t} & 0 \\
0 & e^{-\sqrt{-1} t}
\end{array}\right) \mid t \in \mathbb{R}\right\} .
$$


Hence $S^{2}(1 / 2)$ is represented by $\mathrm{SU}(2) / \mathrm{U}(1)$ as a Riemannian symmetric space. The natural projection

$$
\pi_{1}: S^{3} \rightarrow S^{2}(1 / 2), \quad \pi_{1}(a)=\operatorname{Ad}(a)(\mathbf{k} / 2)
$$

is a Riemannian submersion and defines a principal U(1)-bundle over $S^{2}(1 / 2)$.

Since the Sasaki structure $\left(\eta_{1}, \xi_{1}, \varphi_{1}, g_{1}\right)$ is left invariant, its $D$-homothetic deformation is also left invariant. Hence the elliptic contact space form $\mathcal{M}^{3}(c)$ is identified with $\mathrm{SU}(2)$ with the left invariant contact Riemannian structure:

$$
\begin{gathered}
\eta:=\alpha \eta_{1}, \quad \xi:=\xi_{1} / \alpha, \quad \varphi:=\varphi_{1}, \\
g(X, Y)=\alpha g_{1}(X, Y)+\alpha(\alpha-1) \eta_{1}(X) \eta_{1}(Y), \quad c=4 / \alpha-3 .
\end{gathered}
$$

The Reeb vector field $\xi$ generates a one parameter group of transformations on $\mathcal{M}^{3}(c)$. Since $\xi$ is a Killing vector field, this transformation group acts isometrically on $G$. The transformation group generated by $\xi$ is identified with the following Lie subgroup $K$ of $G$ :

$$
K=\left\{\left(\begin{array}{cc}
e^{\frac{\sqrt{-1} t}{\alpha}} & 0 \\
0 & e^{\frac{-\sqrt{-1} t}{\alpha}}
\end{array}\right) \mid t \in \mathbb{R}\right\} \cong \mathrm{U}(1) .
$$

Furthermore the action of the transformation group generated by $\xi$ corresponds to the natural right action of $K$ on $G$ :

$$
G \times K \rightarrow G ;(a, k) \mapsto a k .
$$

By using the curvature formula due to O'Neill [39], one can see that the orbit space $G / K$ is a 2 -sphere of radius $\sqrt{\alpha} / 2$, namely the constant curvature $(c+3)$-sphere. ( $c f$. Ogiue [38].) The Riemannian metric $g$ is not only $G$-left invariant but also $K$-right invariant. Hence $G \times K$ acts isometrically on $G$. The elliptic contact space form $\mathcal{M}^{3}(c)$ is represented by $G \times K / K=G$ as a naturally reductive homogeneous space. For $c \neq 1, \mathcal{M}^{3}(c)$ has 4-dimensional isometry group.

In particular $g$ is $G$-bi-invariant if and only if $c=1$. In this case $\mathcal{M}^{3}(1)$ is represented by $G \times G / G$ as a Riemannian symmetric space. Note that $\mathcal{M}^{3}(1)$ has 6 -dimensional isometry group.

Now we shall take an orthonormal frame field $\left\{e_{1}, e_{2}, e_{3}\right\}$ of $\mathcal{M}^{3}(c)$ by

$$
e_{1}:=\frac{1}{\sqrt{\alpha}} E_{1}, \quad e_{2}:=\frac{1}{\sqrt{\alpha}} E_{2}, \quad e_{3}:=\frac{1}{\alpha} \xi_{1} .
$$

Then the commutation relations of this basis are

$$
\left[e_{1}, e_{2}\right]=2 e_{3}, \quad\left[e_{2}, e_{3}\right]=\frac{c+3}{2} e_{1}, \quad\left[e_{3}, e_{1}\right]=\frac{c+3}{2} e_{2} .
$$

The Levi-Civita connection $\nabla$ of $\left(\mathcal{M}^{3}(c), g\right)$ is described by

$$
\begin{gathered}
\nabla_{e_{1}} e_{1}=0, \quad \nabla_{e_{1}} e_{2}=e_{3}, \quad \nabla_{e_{1}} e_{3}=-e_{2}, \\
\nabla_{e_{2}} e_{1}=-e_{3}, \quad \nabla_{e_{2}} e_{2}=0, \quad \nabla_{e_{2}} e_{3}=e_{1}, \\
\nabla_{e_{3}} e_{1}=\frac{c+1}{2} e_{2}, \quad \nabla_{e_{3}} e_{2}=-\frac{c+1}{2} e_{1}, \quad \nabla_{e_{3}} e_{3}=0 .
\end{gathered}
$$

The Riemannian curvature tensor field $R$ of $\left(\mathcal{M}^{3}(c), g, \nabla\right)$ is described by

$$
R_{1212}=c, \quad R_{1313}=R_{2323}=1
$$


and the sectional curvatures are:

$$
K_{12}=c, K_{13}=K_{23}=1 \text {. }
$$

The Ricci tensor Ric and the scalar curvature $s$ are computed to be

$$
R_{11}=R_{22}=c+1, R_{33}=2, s=2(c+2) .
$$

5. Parabolic contact space forms. The 3-dimensional Heisenberg group $N^{3}$ is $\mathbb{R}^{3}(x, y, z)$ together with the group structure:

$$
(x, y, z) \cdot\left(x^{\prime}, y^{\prime}, z^{\prime}\right):=\left(x+x^{\prime}, y+y^{\prime}, z+z^{\prime}+\left(x y^{\prime}-x^{\prime} y\right) / 2\right) .
$$

We define a left invariant Riemannian metric $g$ by

$$
g=\frac{d x^{2}+d y^{2}}{4}+\frac{1}{4}\left(d z+\frac{y d x-x d y}{2}\right)^{2} .
$$

Then the Riemannian homogeneous space $\left(N^{3}, g\right)$ has 4-dimensional isometry group. In fact the identity component of the isometry group of $\left(N^{3}, g\right)$ is isomorphic to the semi-direct product $\mathrm{SO}(2) \ltimes N^{3}$. The action of $\mathrm{SO}(2) \ltimes N^{3}$ on $N^{3}$ is

$$
\begin{gathered}
\left(\left[\begin{array}{cc}
\cos \theta & -\sin \theta \\
\sin \theta & \cos \theta
\end{array}\right],\left[\begin{array}{l}
a \\
b \\
c
\end{array}\right]\right) \cdot\left[\begin{array}{l}
x \\
y \\
z
\end{array}\right] \\
=\left[\begin{array}{ccc}
\cos \theta & -\sin \theta & 0 \\
\sin \theta & \cos \theta & 0 \\
\frac{1}{2}(a \sin \theta-b \cos \theta) & \frac{1}{2}(a \cos \theta+b \sin \theta) & 1
\end{array}\right]\left[\begin{array}{l}
x \\
y \\
z
\end{array}\right]+\left[\begin{array}{l}
a \\
b \\
c
\end{array}\right] .
\end{gathered}
$$

The Heisenberg group $\left(N^{3}, g\right)$ is represented by $\mathrm{SO}(2) \ltimes N^{3} / \mathrm{SO}(2)$. This is a naturally reductive homogeneous space representation for $\left(N^{3}, g\right)$. The additive group $(\mathbb{R},+)$ acts isometrically and freely on $N^{3}$ :

$$
N^{3} \times \mathbb{R} \rightarrow N^{3} ; \quad(x, y, z) \cdot a=(x, y, z+a) .
$$

The natural projection $\pi: N^{3} \rightarrow N^{3} / \mathbb{R}=\mathbb{R}^{2}(x, y)$ defines a principal line bundle over $\mathbb{R}^{2}(x, y)$. The metric $g$ induces a flat Riemannian metric $\left(d x^{2}+d y^{2}\right) / 4$ on $\mathbb{R}^{2}(x, y)$. Furthermore $\pi$ is a Riemannian submersion.

Taking a left invariant orthonormal frame field $\mathcal{E}=\left(e_{1}, e_{2}, e_{3}\right)$ :

$$
e_{1}=2 \frac{\partial}{\partial x}-y \frac{\partial}{\partial z}, \quad e_{2}=2 \frac{\partial}{\partial y}+x \frac{\partial}{\partial z}, \quad e_{3}=2 \frac{\partial}{\partial z},
$$

the commutation relations of $\mathcal{E}$ are

$$
\left[e_{1}, e_{2}\right]=2 e_{3},\left[e_{2}, e_{3}\right]=\left[e_{3}, e_{1}\right]=0 .
$$

The dual coframe field $\vartheta=\left(\theta^{1}, \theta^{2}, \theta^{3}\right)$ is given by

$$
\theta^{1}=\frac{1}{2} d x, \theta^{2}=\frac{1}{2} d y, \theta^{3}=\frac{1}{2} d z-\frac{x d y-y d x}{4} .
$$

Note that the 1 -form $\eta:=\theta^{3}$ is a contact form on $N^{3}$. The Levi-Civita connection $\nabla$ of $\left(N^{3}, g\right)$ is given by

$$
\nabla_{e_{1}} e_{2}=-\nabla_{e_{2}} e_{1}=e_{3}, \quad \nabla_{e_{1}} e_{3}=\nabla_{e_{3}} e_{1}=-e_{2}, \quad \nabla_{e_{2}} e_{3}=\nabla_{e_{3}} e_{2}=e_{1}
$$


The Riemannian curvature tensor $R$ of $\left(N^{3}, g\right)$ is described by

$$
R_{1212}=-3, R_{1313}=R_{2323}=1 \text {. }
$$

The sectional curvatures are

$$
K_{12}=-3, \quad K_{13}=K_{23}=1 .
$$

Define an endomorphism field $\varphi$ by

$$
\varphi e_{1}=e_{2}, \varphi e_{2}=-e_{1}, \varphi \xi=0, \quad \xi=e_{3} .
$$

Then $(\eta, \xi, \varphi)$ is a left invariant almost contact structure on $N^{3}$. Since the metric $g$ is related to this almost contact structure by

$$
d \eta(X, Y)=2 g(X, \varphi Y)
$$

Hence $\left(N^{3}, \eta, \xi, \varphi, g\right)$ is a contact Riemannian manifold. Moreover the holomorphic sectional curvature of $N^{3}$ is constantly -3 . From Tanno's classification we then obtain the following result.

Proposition 5.1. The simply connected parabolic contact space form $\mathcal{M}^{3}(-3)$ is isomorphic to the Heisenberg group $N^{3}$.

The Heisenberg group $N^{3}(x, y, z)$ is isomorphic to the following linear Lie group:

$$
\mathbb{H}_{3}(x, y, t)=\left\{\left(\begin{array}{ccc}
1 & y & t \\
0 & 1 & x \\
0 & 0 & 1
\end{array}\right) ; x, y, t \in \mathbb{R}\right\} .
$$

In fact, the mapping $\iota: N^{3} \rightarrow \mathrm{GL}_{3} \mathbb{R}$ defined by

$$
\iota(x, y, z)=\left(\begin{array}{ccc}
1 & y & t \\
0 & 1 & x \\
0 & 0 & 1
\end{array}\right), \quad t=z+\frac{x y}{2}
$$

is a Lie group isomorphism between $N^{3}$ and $\mathbb{H}_{3}$. The homogeneous Sasaki metric on $\mathbb{H}_{3}$ induced by $g$ is written as

$$
g_{\mathbb{H}}=\frac{d x^{2}+d y^{2}}{4}+\frac{1}{4}(d t-y d x)^{2} .
$$

The contact form $\eta$ corresponds to

$$
\eta_{\mathbb{H}}=\frac{1}{2}(d t-y d x) .
$$

Hence the Sasaki manifold $\left(\mathbb{H}_{3}, \eta_{\mathbb{H}}, g_{\mathbb{H}}\right)$ coincides with standard model of $\mathcal{M}^{3}(-3)$. (See Example A in [13], p. 29 and p. 81.)

6. Hyperbolic contact space forms. Let $H^{2}(1 / 2)$ be a hyperbolic 2 -space of constant curvature -4 :

$$
H^{2}(1 / 2)=\left(\left\{(x, y) \in \mathbb{R}^{2} \mid y>0\right\}, \frac{d x^{2}+d y^{2}}{4 y^{2}}\right) .
$$

As is well known the special linear group

$$
G=\mathrm{SL}_{2} \mathbb{R}=\left\{X \in \mathrm{GL}_{2} \mathbb{R} \mid \operatorname{det} g=1\right\} .
$$


acts isometrically and transitively on $H^{2}(1 / 2)$ as linear fractional transformation group. The isotropy subgroup $K$ of $G$ at $(0,1)$ is a rotation group $\mathrm{SO}(2)$. Hence $H^{2}(1 / 2)$ is represented as $H^{2}(1 / 2)=G / K$. It is well known that any element $A \in G$ is decomposed uniquely as

$$
A=\left(\begin{array}{cc}
1 & x \\
0 & 1
\end{array}\right)\left(\begin{array}{cc}
\sqrt{y} & 0 \\
0 & 1 / \sqrt{y}
\end{array}\right)\left(\begin{array}{cc}
\cos \theta & \sin \theta \\
-\sin \theta & \cos \theta
\end{array}\right)
$$

for $x \in \mathbb{R}, y \in \mathbb{R}^{+}, \theta \in S^{1}$. (The Iwasawa decomposition of $A \in \mathrm{SL}_{2} \mathbb{R}$.) In particular $G$ is diffeomorphic to $H^{2} \times S^{1}$. Note that $G$ is not simply connected.

On the Lie algebra $\mathfrak{g}$ of $G$, we shall define an inner product $\langle\cdot, \cdot\rangle$ :

$$
\langle X, Y\rangle=\frac{1}{2} \operatorname{tr}\left({ }^{t} X Y\right), \quad X, Y \in \mathfrak{g} .
$$

The left invariant Riemannian metric $g$ on $G$ induced by $\langle\cdot, \cdot\rangle$ is given explicitly by

$$
g=\frac{d x^{2}+d y^{2}}{4 y^{2}}+\left(d \theta+\frac{d x}{2 y}\right)^{2} .
$$

The Riemannian homogeneous space $(G, g)$ has 4-dimensional isometry group. In fact, $g$ is not only left $G$-invariant but also right $K$-invariant. Hence $G \times K$ acts isometrically and transitively on $G$. Thus $G$ is represented as $G=(G \times K) / K$. This gives a naturally reductive homogeneous space representation for $(G, g)$. Furthermore the natural projection $\pi: G \rightarrow H^{2}(1 / 2)$ is a Riemannian submersion.

Taking an orthonormal frame field $\mathcal{E}=\left(e_{1}, e_{2}, e_{3}\right)$

$$
e_{1}=2 y \frac{\partial}{\partial x}-\frac{\partial}{\partial \theta}, \quad e_{2}=2 y \frac{\partial}{\partial y}, \quad e_{3}=\frac{\partial}{\partial \theta},
$$

then the dual coframe field is given by $\vartheta=\left(\theta^{1}, \theta^{2}, \theta^{3}\right)$ of $\mathcal{E}$ is

$$
\theta^{1}=\frac{d x}{2 y}, \quad \theta^{2}=\frac{d y}{2 y}, \quad \theta^{3}=d \theta+\frac{d x}{2 y} .
$$

The one form $\eta:=-\theta^{3}$ is a left invariant contact form on $G$.

$$
\begin{array}{ccc}
\nabla_{e_{1}} e_{1}=2 e_{2}, & \nabla_{e_{1}} e_{2}=-2 e_{1}-e_{3}, & \nabla_{e_{1}} e_{3}=e_{2}, \\
\nabla_{e_{2}} e_{1}=e_{3} & \nabla_{e_{2}} e_{2}=0, & \nabla_{e_{2}} e_{3}=-e_{1}, \\
\nabla_{e_{3}} e_{1}=e_{2}, & \nabla_{e_{3}} e_{2}=-e_{1}, & \nabla_{e_{3}} e_{3}=0 .
\end{array}
$$

The Riemannian curvature of $G$ is described by

$$
R_{1212}=-7, \quad R_{1313}=R_{2323}=1 .
$$

Hence the sectional curvatures are given by

$$
K_{12}=-7, \quad K_{13}=K_{23}=1 .
$$

We define an endomorphism field $\varphi$ by

$$
\varphi e_{1}=e_{2}, \varphi e_{2}=-e_{1}, \varphi \xi=0, \xi=-e_{3} .
$$

Then $(\eta, \xi, \varphi)$ is an almost contact structure on $G$. The left invariant metric $g$ is related to this almost contact structure by

$$
d \eta(X, Y)=2 g(X, \varphi Y)
$$

Hence $(\xi, \varphi, g)$ is associated to $\eta$. 
Using the above formulas, the following result is easy to check.

Proposition 6.1. The almost contact manifold $(G, \eta ; \xi, \varphi, g)$ is a Sasaki manifold of constant holomorphic sectional curvature -7 .

Again by Tanno's classification we have the following result.

COROLLARY 6.2. Every simply connected hyperbolic contact space form $\mathcal{M}^{3}(c)$ is isomorphic to $\widetilde{\mathrm{SL}_{2}} \mathbb{R}$ up to homothety.

7. Bianchi-Cartan-Vranceanu metrics. Let $\left(M^{3}, g\right)$ be a Riemannian manifold. Then the dimension of the isometry group $I(M, g)$ is at most 6 . In particular $\operatorname{dim} I\left(M^{3}, g\right)$ $=6$ if and only if $\left(M^{3}, g\right)$ is of constant curvature. Furthermore, it is known that there exist no Riemannian 3-manifold with 5-dimensional isometry group (See p. 47, Theorem 3.2 in [33].) If $\operatorname{dim} I\left(M^{3}, g\right)=4$, then the action of isometry group is transitive. L. Bianchi [11], [12] gave a local classification of 3-dimensional homogeneous Riemannian metrics. Furthermore É. Cartan [21] has classified all 4-dimensional isometry groups on Riemannian 3-manifolds (pp. 293-306 in [21]). See also Vranceanu [52]. In [21] and [52], we can see the following 2-parameter family of Riemannian metrics:

$$
\bar{g}_{\lambda, \mu}=\frac{d x^{2}+d y^{2}}{\left\{1+\mu\left(x^{2}+y^{2}\right)\right\}^{2}}+\left(d z+\frac{\lambda}{2} \frac{y d x-x d y}{1+\mu\left(x^{2}+y^{2}\right)}\right)^{2}, \lambda, \mu \in \mathbb{R} .
$$

This 2-parameter family $\left\{\bar{g}_{\lambda, \mu}\right\}$ of Riemannian metrics is called the Bianchi-CartanVranceanu metrics. The metrics as above are defined over the whole 3 -space $\mathbb{R}^{3}$ for $\mu \geq 0$ and over the region $x^{2}+y^{2}<-1 / \mu$ for $\mu<0$. We shall call the homogeneous Riemannian 3-manifolds $\mathfrak{M}^{3}=\mathfrak{M}_{\lambda, \mu}^{3}$ defined by

$$
\mathfrak{M}^{3}=\left(\left\{(x, y, z) \in \mathbb{R}^{3} \mid 1+\mu\left(x^{2}+y^{2}\right)>0\right\}, \bar{g}_{\lambda, \mu}\right)
$$

the Bianchi-Cartan-Vranceanu spaces (BCV-spaces for short). It is known that a BCVspace $\mathfrak{M}^{3}$ is isometric to the following homogeneous Riemannian 3-manifolds:

- If $\mu=\lambda=0$, then $\mathfrak{M} \cong \mathbb{E}^{3}$ (Euclidean 3-space),

- If $\mu=0, \lambda \neq 0$, then $\mathfrak{M} \cong N^{3}$ (Heisenberg group),

- If $\mu>0, \lambda \neq 0$, then $\mathfrak{M} \cong \mathrm{SU}(2) \backslash\{\infty\}$ with left invariant metric,

- If $\mu<0, \lambda \neq 0$, then $\mathfrak{M} \cong \widetilde{\mathrm{SL}}_{2} \mathbb{R}$ with left invariant metric,

- If $\mu>0, \lambda=0$, then $\mathfrak{M} \cong S^{2}\left(\sqrt{\mu^{-1}} / 2\right) \backslash\{\infty\} \times \mathbb{R}$,

- If $\mu<0, \lambda=0$, then $\mathfrak{M} \cong H^{2}\left(\sqrt{-\mu^{-1}} / 2\right) \times \mathbb{R}$,

- If $4 \mu-\lambda^{2}=0$ then $\mathfrak{M} \cong S^{3}(2 / \lambda) \backslash\{\infty\}$.

Here $S^{2}(r)$ and $H^{2}(r)$ denote the 2-sphere of radius $r$ and the hyperbolic 2-space of radius $r$.

In this section we shall study canonical almost contact structures of BCV-spaces.

Take an orthonormal frame field $\mathcal{E}=\left(e_{1}, e_{2}, e_{3}\right)$ :

$$
e_{1}=\left\{1+\mu\left(x^{2}+y^{2}\right)\right\} \frac{\partial}{\partial x}-\frac{\lambda y}{2} \frac{\partial}{\partial z}, \quad e_{2}=\left\{1+\mu\left(x^{2}+y^{2}\right)\right\} \frac{\partial}{\partial y}+\frac{\lambda x}{2} \frac{\partial}{\partial z}, \quad e_{3}=\frac{\partial}{\partial z}
$$


Then the dual coframe field $\vartheta=\left(\theta^{1}, \theta^{2}, \theta^{3}\right)$ is given by

$$
\theta^{1}=\frac{d x}{1+\mu\left(x^{2}+y^{2}\right)}, \quad \theta^{2}=\frac{d y}{1+\mu\left(x^{2}+y^{2}\right)}, \quad \theta^{3}=d z+\frac{\lambda}{2} \frac{y d x-x d y}{1+\mu\left(x^{2}+y^{2}\right)} .
$$

Note that the one-form $\eta:=\theta^{3}$ is a contact form on $\mathfrak{M}^{3}$ if and only if $\lambda \neq 0$. The connection 1-forms $\left\{\omega_{j}{ }^{i}\right\}$ defined by $d \theta^{i}+\sum \omega_{j}{ }^{i} \wedge \theta^{j}=0$ relative to $\vartheta$ are given by

$$
\left(\omega_{j}^{i}\right)=\left(\begin{array}{ccc}
0 & -2 \mu\left(y \theta^{1}-x \theta^{2}\right)+\frac{\lambda}{2} \theta^{3} & \frac{\lambda}{2} \theta^{2} \\
2 \mu\left(y \theta^{1}-x \theta^{2}\right)-\frac{\lambda}{2} \theta^{3} & 0 & -\frac{\lambda}{2} \theta^{1} \\
-\frac{\lambda}{2} \theta^{2} & \frac{\lambda}{2} \theta^{1} & 0
\end{array}\right) .
$$

In covariant derivative fashion, the Levi-Civita connection $\bar{\nabla}$ of $\mathfrak{M}$ is described by the formulae:

$$
\begin{gathered}
\bar{\nabla}_{e_{1}} e_{1}=2 \mu y e_{2}, \quad \bar{\nabla}_{e_{1}} e_{2}=-2 \mu y e_{1}+\frac{\lambda}{2} e_{3}, \quad \bar{\nabla}_{e_{1}} e_{3}=-\frac{\lambda}{2} e_{2}, \\
\bar{\nabla}_{e_{2}} e_{1}=-2 \mu x e_{2}-\frac{\lambda}{2} e_{3}, \quad \bar{\nabla}_{e_{2}} e_{2}=2 \mu x e_{1}, \quad \bar{\nabla}_{e_{2}} e_{3}=\frac{\lambda}{2} e_{1}, \\
\bar{\nabla}_{e_{3}} e_{1}=-\frac{\lambda}{2} e_{2}, \quad \bar{\nabla}_{e_{3}} e_{2}=\frac{\lambda}{2} e_{1}, \quad \bar{\nabla}_{e_{3}} e_{3}=0 . \\
{\left[e_{1}, e_{2}\right]=-2 \mu y e_{1}+2 \mu x e_{2}+\lambda e_{3}, \quad\left[e_{2}, e_{3}\right]=\left[e_{3}, e_{1}\right]=0 .}
\end{gathered}
$$

The curvature forms $\Omega$ relative to $\vartheta$ are given by

$$
\Omega_{2}{ }^{1}=\left(4 \mu-\frac{3 \lambda^{2}}{4}\right) \theta^{1} \wedge \theta^{2}, \quad \Omega_{3}{ }^{1}=\frac{\lambda^{2}}{4} \theta^{1} \wedge \theta^{3}, \quad \Omega_{3}{ }^{2}=\frac{\lambda^{2}}{4} \theta^{2} \wedge \theta^{3},
$$

hence the curvature tensor $\bar{R}$ is described by the formula:

$$
\bar{R}_{1212}=4 \mu-\frac{3}{4} \lambda^{2}, \quad \bar{R}_{1313}=\bar{R}_{2323}=\frac{\lambda^{2}}{4} .
$$

The Ricci tensor $\overline{\text { Ric }}$ of $\mathfrak{M}$ is given by

$$
\bar{R}_{11}=\bar{R}_{22}=4 \mu-\lambda^{2}, \quad \bar{R}_{33}=\frac{\lambda^{2}}{2} .
$$

Hence the scalar curvature $\bar{s}$ is $\bar{s}=8 \mu-\lambda^{2} / 2$.

REMARK 7.1. The geodesics of $\mathfrak{M}^{3}$ are calculated by Sitzia [42]. See also Borghero and Caddeo [17].

Define an endomorphism field $\varphi$ by $\varphi e_{1}=e_{2}, \quad \varphi e_{2}=-e_{1}, \quad \varphi e_{3}=0$ and define $\xi:=e_{3}$. Then it is easy to check that $\left(\eta, \xi, \varphi, \bar{g}_{\lambda, \mu}\right)$ is an almost contact structure of $\mathfrak{M}^{3}$. In particular if $\lambda \neq 0,\left(\varphi, \xi, \bar{g}_{\lambda, \mu}\right)$ is the associated almost contact structure of $\eta$ up to a constant multiple. More precisely the exterior derivative $d \eta$ is related to $\varphi$ by

$$
d \eta(X, Y)=\lambda \bar{g}(X, \varphi Y), \quad X, Y \in \mathfrak{X}\left(\mathfrak{M}^{3}\right) .
$$

This almost contact structure satisfies the following:

$$
\left(\bar{\nabla}_{X} \varphi\right) Y=\frac{\lambda}{2}\{\bar{g}(X, Y) \xi-\eta(Y) X\}, \quad \bar{\nabla}_{X} \xi=-\frac{\lambda}{2} \varphi X .
$$

These formulae show that $\xi$ is a Killing vector field on $\mathfrak{M}^{3}$. Moreover $\mathfrak{M}^{3}$ is an almost contact manifold of constant holomorphic sectional curvature $c=4 \mu-3 \lambda^{2} / 4$. In particular if $\lambda \neq 0$, then it is a $(\lambda / 2)$-Sasaki manifold of constant holomorphic sectional curvature 
$\bar{c}=4 \mu-3 \lambda^{2} / 4$. Namely $\mathfrak{M}^{3}$ is homothetic to a Sasakian manifold of constant holomorphic sectional curvature. (See Proposition 2.1.)

The vector field $\xi$ is Killing, complete and it generates a one-parameter group of isometries on $\mathfrak{M}^{3}$. Furthermore this group action is simply transitive. The orbit space $\tilde{\mathfrak{M}}^{2}=\mathfrak{M}^{3} / \xi$ is

$$
\tilde{\mathfrak{M}}^{2}=\left(\left\{(x, y) \in \mathbb{R}^{2} \mid 1+\mu\left(x^{2}+y^{2}\right)>0\right\}, \frac{d x^{2}+d y^{2}}{\left\{1+\mu\left(x^{2}+y^{2}\right)\right\}^{2}}\right) .
$$

The natural projection $\pi: \mathfrak{M}^{3} \rightarrow \tilde{\mathfrak{M}}^{2}$ defined by $\pi(x, y, z)=(x, y)$ is a Riemannian submersion with totally geodesic fibres. The base space $\tilde{\mathfrak{M}}^{2}$ is of constant curvature $4 \mu$.

In case $\lambda \neq 0$, under the normalization:

$$
\hat{\eta}:=\frac{\lambda}{2} \eta, \quad \hat{\xi}:=\frac{2}{\lambda} \xi, \quad \hat{\varphi}:=\varphi, \quad \hat{g}=\frac{\lambda^{2}}{4} \bar{g},
$$

the almost contact manifold $\hat{\mathfrak{M}}^{3}=\left(\mathfrak{M}^{3}, \hat{\eta} ; \hat{\xi}, \hat{\varphi}, \hat{g}\right)$ is a simply connected Sasaki manifold of constant holomorphic sectional curvature $\hat{c}:=-3+16 \mu / \lambda^{2}$. Under this normalization, the base space $\hat{\mathfrak{M}}^{2}$ of the fibering $\pi: \hat{\mathfrak{M}}^{3}(\hat{c}) \rightarrow \hat{\mathfrak{M}}^{2}$ is a Riemannian 2-manifold of constant curvature $16 \mu / \lambda^{2}$.

In particular if $\mu \leq 0, \hat{\mathfrak{M}}^{3}$ is complete and simply connected and hence a parabolic or hyperbolic contact space form.

Now we can give an explicit description of a compatible almost contact structure on BCV-spaces.

(1) $\mu=\lambda=0$ : In this case $\mathfrak{M}^{3}$ is a Euclidean 3 -space $\mathbb{E}^{3}$.

(2) $\mu=0, \lambda \neq 0$ : Without loss of generality, we may assume $(\lambda, \mu)=(1,0)$.

Proposition 7.2. The homogeneous Riemannain manifold $\mathfrak{M}_{1,0}^{3}$ is homothetic to a parabolic contact space form. More precisely it is homothetic to the Heisenberg group $N^{3}$ with left invariant Sasaki structure. The metrics $\bar{g}_{1,0}$ and $g$ in (5.1) are related by $g=\bar{g}_{1,0} / 4$.

(3) $\mu<0, \lambda \neq 0$ : In this case we may assume $(\lambda, \mu)=(2,-1)$. Note that, in this case, $\mathfrak{M}_{2,-1}^{3}=\hat{\mathfrak{M}}_{2,-1}^{3}$.

COROLlary 7.3. The homogeneous Riemannian manifold $\mathfrak{M}_{2,-1}^{3}$ admits a left invariant Sasaki structure of constant holomorphic sectional curvature -7 . More precisely $\mathfrak{M}_{2,-1}^{3}$ can be identified with $\left(\widetilde{\mathrm{SL}_{2}} \mathbb{R}, g\right)$ in section 6 .

(4) $\mu>0, \lambda \neq 0$ : In this case, $\hat{\mathfrak{M}}^{3}$ is an open dense subset of an elliptic contact space form. In fact, elliptic contact space forms are topologically 3 -spheres, and $\hat{\mathfrak{M}}^{3}$ is diffeomorphic to $\mathbb{R}^{3}$. The base space $\hat{\mathfrak{M}}^{2}$ of the fibering $\pi: \overline{\mathcal{M}}^{3}(\hat{c}) \rightarrow \hat{\mathfrak{M}}^{2}$ is a Riemannian 2-manifold of constant curvature $16 \mu / \lambda^{2}$.

Proposition 7.4. The universal covering manifold of $\mathfrak{M}_{\lambda, \mu}^{3}$ with $\lambda \neq 0, \mu>0$ is homothetic to a simply connected elliptic contact space form $\mathcal{M}^{3}(c)=\mathrm{SU}(2), c>-3$. In particular $\mathfrak{M}_{\lambda, \mu}^{3}$ is of constant curvature if and only if $\lambda^{2}=4 \mu$.

(5) $\mu<0, \lambda=0$ : In this case $\mathfrak{M}^{3}$ is a Riemannian product of the hyperbolic 2-space $H^{2}\left(\sqrt{-\mu^{-1}} / 2\right)$ of constant curvature $4 \mu$ (or more explicitly the open disk $\mathfrak{D}(1 / \sqrt{-\mu})$ 
with radius $1 / \sqrt{-\mu}$ centered at the origin together with the metric:

$$
\left.\frac{d x^{2}+d y^{2}}{\left\{1+\mu\left(x^{2}+y^{2}\right)\right\}^{2}}\right)
$$

and a real line $\mathbb{R}(z)$. Hence the base space $\tilde{\mathfrak{M}}^{2}$ coincides with $\mathfrak{D}(1 / \sqrt{-\mu})$ and the projection $\pi$ is nothing but the projection $\mathfrak{M}^{3} \rightarrow \mathfrak{D}(1 / \sqrt{-\mu})$. Let us denote by $J$ the complex structure of $\mathfrak{D}(1 / \sqrt{-\mu})$. Then the almost contact structure is given by

$$
\varphi\left(X, f \frac{d}{d t}\right)=(J X, 0), \quad X \in \mathfrak{X}(\mathfrak{D}(1 / \sqrt{-\mu})), \quad f \in C^{\infty}\left(\mathfrak{M}^{3}\right) .
$$

Obviously this almost contact structure is non contact and satisfies $\bar{\nabla} \varphi=0, \bar{\nabla} \xi=0$. The one form $\eta=d z$ defines a trivial flat connection on the line bundle $\mathfrak{M}^{3}=\mathfrak{D}(1 / \sqrt{-\mu}) \times \mathbb{R}$.

(6) $\mu>0, \lambda=0$ : In this case $\mathfrak{M}^{3}$ is a Riemannian product of a plane $\mathbb{R}^{2}(x, y)$ with constant positive curvature metric:

$$
\frac{d x^{2}+d y^{2}}{\left\{1+\mu\left(x^{2}+y^{2}\right)\right\}^{2}}
$$

and a real line $\mathbb{R}(z)$. As is well known this metric on $\mathbb{R}^{2}$ is induced from the metric of the 2-sphere

$$
S^{2}\left(\sqrt{\mu^{-1}} / 2\right)=\left\{(x, y, t) \in \mathbb{R}^{3} \mid x^{2}+y^{2}+t^{2}=\mu^{-1} / 4\right\}
$$

via the stereographic projection with respect to the north pole.

Hence the base space $\tilde{\mathfrak{M}}^{2}$ coincides with $\mathbb{R}^{2}$ with metric (7.5) and the projection $\pi$ is nothing but the projection $\mathfrak{M}^{3} \rightarrow \mathbb{R}^{2}$. Let us denote by $J$ the complex structure of $\mathbb{R}^{2}$. Then the almost contact structure is given by

$$
\varphi\left(X, f \frac{d}{d t}\right)=(J X, 0), \quad X \in \mathfrak{X}\left(\mathbb{R}^{2}\right), \quad f \in C^{\infty}\left(\mathfrak{M}^{3}\right) .
$$

Obviously this almost contact structure is non contact and satisfies $\bar{\nabla} \varphi=0, \bar{\nabla} \xi=0$. The one form $\eta=d z$ defines a trivial flat connection on the line bundle $\mathfrak{M}^{3}=\mathbb{R}^{2} \times \mathbb{R}$.

8. Surfaces with parallel second fundamental form in $\mathfrak{M}_{\lambda, \mu}^{3}$. Let $\mathfrak{M}_{\lambda, \mu}^{3}$ be a BCV-space with constant holomorphic sectional curvature $c=4 \mu-3 \lambda^{2} / 4$.

We shall determine all surfaces in $\mathfrak{M}_{\lambda, \mu}^{3}$ with parallel second fundamental form.

First we shall recall the classification of surfaces with parallel second fundamental form in Riemannian 3-space forms.

Without loss of generality we may restrict our attention to surfaces in $\mathbb{E}^{3}$, unit 3-sphere $S^{3}$ and unit hyperbolic 3 -space $H^{3}$. It is well known that surfaces with parallel second fundamental form in $\mathbb{E}^{3}$ are planes (totally geodesic surfaces), spheres (totally umbilic surfaces) or circular cylinders ( $c f$. [31]).

In hyperbolic 3 -space $H^{3}$, surfaces with parallel second fundamental form are totally geodesic hyperbolic 2-spaces, totally umbilic 2-spheres or equidistant surfaces around a geodesic.

Finally in $S^{3}$, surfaces with parallel second fundamental form are great spheres (totally geodesic surfaces), small spheres (totally umbilic surfaces) or Hopf cylinders over a Riemannian circle in the base space $S^{2}(1 / 2)$ of the Hopf fibering $S^{3} \rightarrow S^{2}(1 / 2)$ (cf. 
[1] and [44]). Here Riemannian circles in $S^{2}(1 / 2)$ are curves in $S^{2}(1 / 2)$ with constant curvature.

REMARK 8.1 ( $B$-scroll). A curve in $S^{3}(1)$ of constant torsion \pm 1 whose geodesic curvature is a polynomial of the arc length parameter is called a twisted spherical polynomial spiral. In particular if the geodesic curvature is constant, such a curve is called a twisted spherical spiral. The second author showed in [23] that a $B$-scroll of a twisted spherical polynomial spiral whose geodesic curvature is of degree $k$ is a flat surface and its $(k+1)$-th covariant derivative of the second fundamental form vanishes. In particular the $B$-scroll of a twisted spherical spiral has parallel second fundamental form. One can check that a $B$-scroll of a twisted spherical spiral is a Hopf cylinder (torus) over a Riemannian circle in $S^{2}(1 / 2)$.

Since parallel surfaces in simply connected Riemannian 3-space forms are classified, we may restrict our investigation to $\mathfrak{M}_{\lambda, \mu}^{3}$ with $4 \mu-\lambda^{2} \neq 0$. In this section we shall investigate such surfaces in $\mathfrak{M}_{\lambda, \mu}^{3}$ with $\lambda \neq 0,4 \mu-\lambda^{2} \neq 0$.

Let $S$ be a surface in $\mathfrak{M}^{3}$ with unit normal vector field $N$. Denote by $\nabla$ the Levi-Civita connection of $M$ induced by $\bar{\nabla}$. Then the second fundamental form $h$ of $M$ is defined by the Gauss formula:

$$
\bar{\nabla}_{X} Y=\nabla_{X} Y+h(X, Y), \quad X, Y \in \mathfrak{X}(S) .
$$

For any $X, Y, Z \in \mathfrak{X}(S)$, the normal component of the curvature $\bar{R}(X, Y) Z$ satisfies the Codazzi equation:

$$
(\bar{R}(X, Y) Z)^{\perp}=\left(\nabla_{X} h\right)(Y, Z)-\left(\nabla_{Y} h\right)(X, Z) .
$$

Here the covariant derivative $\nabla h$ is defined by

$$
\left(\nabla_{X} h\right)(Y, Z)=\nabla_{X}^{\perp} h(Y, Z)-h\left(\nabla_{X} Y, Z\right)-h\left(Y, \nabla_{X} Z\right)
$$

for all $X, Y, Z \in \mathfrak{X}(M)$. Here $\nabla^{\perp}$ denotes the normal connection of $S$ in $\mathfrak{M}^{3}$.

A surface $S$ is said to be parallel if $\nabla h=0$.

TheOREM 8.2. Let $\mathfrak{M}_{\lambda, \mu}^{3}$ be the BCV-space with $4 \mu-\lambda^{2} \neq 0$.

(i) If $\lambda \neq 0$, then the only parallel surfaces in $\mathfrak{M}_{\lambda, \mu}^{3}$ are Hopf cylinders over Riemannian circles in $\tilde{\mathfrak{M}}^{2}$.

(ii) If $\lambda=0$, then the only parallel surfaces in Riemannian symmetric space $\mathfrak{M}_{0, \mu}^{3}$ with $\mu \neq 0$ are totally geodesic leaves and Hopf cylinders over Riemannian circles in $\tilde{\mathfrak{M}}^{2}$.

Here Riemannian circles in $\tilde{\mathfrak{M}}^{2}$ are curves in $\tilde{\mathfrak{M}}^{2}$ with constant geodesic curvature.

Proof. Let $\left\{X_{1}, X_{2}, X_{3}\right\}$ be an orthonormal frame field of $S$ such that $X_{3}=N$. Denote by $\left\{\omega^{1}, \omega^{2}, \omega^{3}\right\}$ the dual coframe field to $\left\{X_{i}\right\}$. We can write, using the notaions of the previous section, $\omega^{3}=p \theta^{1}+q \theta^{2}+r \theta^{3}$. Since $\left\{\omega^{i}\right\}$ is orthonormal, $p^{2}+q^{2}+r^{2}=1$. The vector fields $u_{1}=r e_{1}-p e_{3}, u_{2}=r e_{2}-q e_{3}$ are tangent to $S$. In particular if $r \neq 0$, $\left\{u_{1}, u_{2}\right\}$ is a (local) frame field on $S$. By direct computations using (7.4) we obtain the following:

LEMma 8.3. The normal components of curvature tensor $\bar{R}$ are described by

$$
\left(\bar{R}\left(u_{1}, u_{2}\right) u_{1}\right)^{\perp}=q r^{3}\left(\lambda^{2}-4 \mu\right) N, \quad\left(\bar{R}\left(u_{1}, u_{2}\right) u_{1}\right)^{\perp}=-p r^{3}\left(\lambda^{2}-4 \mu\right) N .
$$


Assume that the second fundamental form $h$ of $S$ is parallel. Then the Codazzi equation implies:

$$
\left(\bar{R}\left(u_{1}, u_{2}\right) u_{1}\right)^{\perp}=\left(\nabla_{u_{1}} h\right)\left(u_{2}, u_{1}\right)-\left(\nabla_{u_{1}} h\right)\left(u_{1}, u_{1}\right)=0 .
$$

Similarly we have

$$
\left(\bar{R}\left(u_{2}, u_{1}\right) u_{2}\right)^{\perp}=0 .
$$

First we consider the case $r \neq 0$.

CASE I, $r \neq 0$ : The preceding lemma implies $p=q=0$, since we assumed $\lambda^{2}-4 \mu \neq 0$. Hence $\omega^{3}=\theta^{3}$. Namely $S$ is an integral surface of the distribution $D$ defined by $\eta=0$.

Subcase (i), $\lambda=0$ : In this case $S$ is an integral surface of the distribution $D$ defined by $d z=0$. Hence $S$ is a leaf $\tilde{\mathfrak{M}}^{2} \times\left\{z_{0}\right\}$ for some $z_{0}$. Note that this leaf is totally geodesic.

Subcase (ii), $\lambda \neq 0$ : Since $\eta$ in this case is a contact form, there are no integral surfaces of $D$. This implies that this case cannot occur.

CASE II, $r=0$ : In this case $\omega^{3}$ has the form $\omega^{3}=p \theta^{1}+q \theta^{2}$. Since $p^{2}+q^{2}=1$, we may write $p=\cos \phi, q=\sin \phi$. In addition, the orthonormal vector fields $v_{1}=q e_{1}-p e_{2}, v_{2}=$ $e_{3}$ give an orthonormal frame field tangent to $S$.

Next by using (7.2), we have

$$
\begin{gathered}
\bar{\nabla}_{v_{1}} v_{1}=v_{1}(q) e_{1}-v_{1}(p) e_{2}+2 \mu(p x+q y) N, \quad \bar{\nabla}_{v_{1}} v_{2}=-\frac{\lambda}{2} N, \\
\bar{\nabla}_{v_{2}} v_{1}=-\frac{\lambda}{2} N+\left(v_{2}(q) e_{1}-v_{2}(p) e_{2}\right), \quad \bar{\nabla}_{v_{2}} v_{2}=0 .
\end{gathered}
$$

By the symmetry of second fundamental form, we get $p v_{2}(q)-q v_{2}(p)=0$. Equivalently we have $v_{2}(\phi)=0$.

By the Gauss formula we obtain the induced connection and the second fundamental form:

$$
\begin{gathered}
\nabla_{v_{1}} v_{1}=0, \quad \nabla_{v_{1}} v_{2}=0, \quad \nabla_{v_{2}} v_{2}=0 \\
h\left(v_{1}, v_{1}\right)=\left\{v_{1}(\phi)+2 \mu(p x+q y)\right\} N, \quad h\left(v_{1}, v_{2}\right)=-\frac{\lambda}{2} N, \quad h\left(v_{2}, v_{2}\right)=0 .
\end{gathered}
$$

The mean curvature vector field $\mathbb{H}$ of $S$ defined by

$$
\mathbb{H}=\frac{1}{2} \operatorname{tr} h
$$

is computed as

$$
\mathbb{H}=H N, \quad 2 H=v_{1}(\phi)+2 \mu(p x+q y) .
$$

Since $h$ is parallel, the mean curvature $H$ is constant. Furthermore (8.3) implies that $S$ is flat. Moreover (8.3) implies that we can take $z$ as a (local) coordinate of $S$ and hence there exists a local coordinate system $(t, z)$ such that

$$
\frac{\partial}{\partial t}=v_{1} \text {. }
$$

Thus the equation $v_{2}(\phi)=0$ implies that $\phi$ depends only on $t$. With respect to this coordinate system $(t, z)$, the constancy of $H$ is rewritten as

$$
\frac{d \phi}{d t}=2\{H-\mu(\cos \phi(t) x(t)+\sin \phi(t) y(t))\}, \quad H \in \mathbb{R} .
$$


It is easy to see that $S$ is generated by two coordinate curves. The $z$-coordinate curves are geodesics and integral curves of the vector field $e_{3}$. The $t$-coordinate curves are horizontal curves of curvature $2 H$. Let us denote by $\gamma(t)$ the $t$-coordinate curve in $\mathfrak{M}^{3}$. Then it is easy to see that $\gamma$ is a Frenet curve of osculating order at most 3. The principal normal vector field of $\gamma$ is the restriction of $N$ on $\gamma$. The binormal vector field $B$ of $\gamma$ is $\pm e_{3}$. We can assume $B=e_{3}$. The Frenet-Serret formulas of $\gamma$ are given by

$$
\bar{\nabla}_{\gamma^{\prime}}(T, N, B)=(T, N, B)\left(\begin{array}{ccc}
0 & -2 H & 0 \\
2 H & 0 & -\frac{\lambda}{2} \\
0 & \frac{\lambda}{2} & 0
\end{array}\right) .
$$

This implies that $t$-coordinate curves are of constant torsion $\lambda / 2$. Hence $t$-coordinate curves are horizontal curves of curvature $2 H$. (See [2] [28], [30].)

Let us denote by $\tilde{\gamma}(t)=(x(t), y(t))$ the projection of $\gamma$ to $\tilde{\mathfrak{M}}^{2}$, namely $\tilde{\gamma}=\pi \circ \gamma$. One can check that $\tilde{\gamma}(t)$ is of constant curvature $2 H$. Equivalently $t$-coordinate curves are horizontal lifts of Riemannian circles of curvature $2 H$ in $\tilde{\mathfrak{M}}^{2}$. (In particular the case $H=0, \tilde{\gamma}$ is a geodesic in $\tilde{\mathfrak{M}}^{2}$.)

Recall that the surface $S$ is parameterized by $t$ and $z$. And the $t$-coordinate curves are horizontal lifts of Riemannian circles in the base space with curvature $2 H$. Thus we conclude that $S$ is a Hopf cylinder over a Riemannian circle of curvature $2 H$. In particular, $S$ is parameterized by $(x(t), y(t), z)$. We finish this paper by considering these Riemannian circles in more detail.

From the definition of $v_{1}$, we notice that

$$
\frac{d x}{d t}=\sin \phi(t) f(t), \quad \frac{d y}{d t}=-\cos \phi(t) f(t), \quad f(t):=1+\mu\left(x(t)^{2}+y(t)^{2}\right) .
$$

Here $\phi(t)$ is the solution to (8.6). The equation (8.8) says $t$ is the arc length parameter of $\tilde{\gamma}$.

The function $f(t)$ satisfies the following ordinary differential equation:

$$
\frac{d}{d t} \log |f(t)|=2 \mu\{x(t) \sin \phi(t)-y(t) \cos \phi(t)\} .
$$

On the other hand, differentiating (8.6),

$$
\frac{d^{2} \phi}{d t^{2}}(t)=\frac{d \phi}{d t}(t) \frac{d}{d t} \log |f(t)|
$$

If $\phi^{\prime}=0$, then from (8.8) we obtain that $\tilde{\gamma}$ is a straight line. So we assume that $\phi^{\prime} \neq 0$. Comparing (8.9) and (8.10), we have

$$
f(t)=\alpha \phi^{\prime}(t), \quad \alpha \in \mathbb{R} .
$$

Hence we obtain the following explicit expression for $\tilde{\gamma}$ :

$$
(x(t), y(t))=\left(-\alpha \cos \phi(t)+x_{0},-\alpha \sin \phi(t)+y_{0}\right) .
$$

Therefore the curve is a circle centered at $\left(x_{0}, y_{0}\right)$.

COROLlary 8.4. All parallel surfaces in contact space forms $\mathcal{M}^{3}(c), c \neq 1$ are Hopf cylinders over Riemannian circles in the base Riemannian space form $\tilde{\mathcal{M}}^{2}(c+3)$ of constant curvature $(c+3)$. 
Note that Corollary 8.4 implies the nonexistence of totally geodesic surfaces and extrinsic spheres-i.e., totally umbilical surfaces with parallel mean curvature vector fields, in contact 3 -space forms with $c \neq 1$.

REMARK 8.5. In the $\mathrm{SL}_{2} \mathbb{R}$ case, Hopf cylinders coincide with rotational surfaces in the sense of Kokubu [34].

REMARK 8.6. The second named author classified hypersurfaces in Riemannian space forms with higher order parallel second fundamental form [22], [23] and [24]. In particular all the higher order parallel surfaces in $S^{3}$ are classified as follows:

Theorem 8.7 ([23], [24]). Let $M$ be a higher order parallel surface of $S^{3}(1)$. Then either $M$ is an open part of a 2-sphere of $S^{3}$, or of a Hopf cylinder over a spherical curve in $S^{2}(1 / 2)$ whose geodesic curvature is a polynomial function of the arc length parameter.

Based on this result and Theorem 8.2, the following conjecture naturally arises:

Conjecture. A higher order parallel surface in $\mathfrak{M}_{\lambda, \mu}^{3}$ with $4 \mu-\lambda^{2} \neq 0$ is a Hopf cylinder over a curve in the base space $\tilde{\mathfrak{M}}^{2}(4 \mu)$ whose geodesic curvature is a polynomial function of the arc length parameter.

\section{References}

[1] E. Backes and H. Reckziegel, On symmetric submanifolds of spaces of constant curvature, Math. Ann. 263 (1983), 419-433.

[2] C. Baikoussis and D. E. Blair, On Legendre curves in contact 3-manifolds, Geom. Dedicata 49 (1994), 135-142.

[3] M. Barros, Willmore tori in non-standard 3-sphere, Math. Proc. Cambridge Phil. Soc. 121 (1997), 321-324.

[4] M. Belkhelfa and F. Dillen, Parallel surfaces in Heisenberg space, in: Differential Geometry in honor of Radu Rosca, KU Brussels, to appear.

[5] M. Belkhelfa, F. Dillen and J. Inoguchi, Parallel surfaces in the real special linear group $S L(2, \mathbb{R})$, Bull. Austral. Math. Soc. 65 (2002), 183-189.

[6] J. Berndt, Über Untermannigfaltigkeiten von komplexen Raumformen, Ph.D. Thesis, Universität zu Köln, 1989.

[7] J. Berndt, Real hypersurfaces with constant principal curvatures in complex space forms, in: Geometry and Topology of Submanifolds, III, M. Boyom, J. M. Morvan and L. Verstraelen (eds.), World Scientific, 10-19.

[8] J. Berndt and L. Vanhecke, Geometry of weakly symmetric spaces, J. Math. Soc. Japan 48 (1996), 745-760.

[9] V. N. Berestovskiŭ and D. E. Vol'per, A class of $U(n)$-invariant Riemannian metrics on manifolds diffeomorphic to odd-dimensional spheres, Sibirsk. Mat. Zh. 34 (1993), 24-32 (in Russian); English transl., Siberian Math. J. 34 (1993), 612-619.

[10] A. L. Besse, Einstein Manifolds, A Series of Modern Surveys in Math. 10, Springer, 1987.

[11] L. Bianchi, Lezioni di geometrie differenziale, E. Spoerri Librao-Editore, 1894.

[12] L. Bianchi, Lezioni sulla teoria dei gruppi continui e finiti di trasformazioni, Ed. Zanichelli, 1928. 
[13] D. E. Blair, Contact Manifolds in Riemannian Geometry, Lecture Notes in Math. 509, Springer, 1976.

[14] D. E. Blair and L. Vanhecke, Symmetries and $\varphi$-symmetric spaces, Tôhoku Math. J. 39 (1987), 373-383.

[15] D. E. Blair and L. Vanhecke, New characterizations of $\varphi$-symmetric spaces, Kōdai Math. J. 10 (1987), 102-107.

[16] W. M. Boothby and H. C. Wang, On contact manifolds, Ann. Math. 68 (1958), 721-734.

[17] F. Borghero and R. Caddeo, Une structure de séparabilité et géodésiques dans les huit géométries tridimensionalles de Thurston, Rend. Mat. Appl. (7) 9 (1989), 607-624.

[18] R. Caddeo, P. Piu and A. Ratto, $S O(2)$-invariant minimal and constant mean curvature surfaces in 3-dimensional homogeneous spaces, Manuscripta Math. 87 (1995), 1-12.

[19] R. Caddeo, P. Piu and A. Ratto, Rotational surfaces in $\mathrm{H}_{3}$ with constant Gauss curvature, Boll. Un. Mat. Ital. B (7) 10 (1996), 341-357.

[20] G. Calvaruso, D. Perrone and L. Vanhecke, Homogeneity on three-dimensional contact metric manifolds, Israel J. Math. 114 (1999), 301-321.

[21] É. Cartan, Leçons sur la Géométrie des Espaces de Riemann, Gauthier-Villars, Paris, 1928.

[22] F. Dillen, The classification of hypersurfaces of a Euclidean space with parallel higher order fundamental form, Math. Z. 203 (1990), 635-643.

[23] F. Dillen, Hypersurfaces of a real space form with parallel higher order fundamental form, Soochow J. Math. 18 (1992), 321-338.

[24] F. Dillen, Sur les hypersurfaces parallèles d'ordre supérieur, C. R. Acad. Sc. Paris 311 (1990), 185-187.

[25] N. Ejiri, A generalization of minimal cones, Trans. Amer. Math. Soc. 276 (1983), 347-360.

[26] D. Ferus, Symmetric submanifolds of Euclidean space, Math. Ann. 247 (1980), 81-93.

[27] Y. Hatakeyama, Some notes on differentiable manifolds with almost contact structures, Tôhoku Math. J. 15 (1963), 176-181.

[28] J. Inoguchi, T. Kumamoto, N. Ohsugi and Y. Suyama, Differential geometry of curves and surfaces in 3-dimensional homogeneous spaces I, Fukuoka Univ. Sci. Rep. 29 (1999), $155-182$.

[29] J. Inoguchi, T. Kumamoto, N. Ohsugi and Y. Suyama, Differential geometry of curves and surfaces in 3-dimensional homogeneous spaces II, Fukuoka Univ. Sci. Rep. 30 (2000), $17-47$.

[30] J. Inoguchi, T. Kumamoto, N. Ohsugi and Y. Suyama, Differential geometry of curves and surfaces in 3-dimensional homogeneous spaces III, Fukuoka Univ. Sci. Rep. 30 (2000), $130-161$.

[31] V. F. Kagan, The Fundamentals of the Theory of Surfaces in Tensor Representation II, Surfaces in Spaces, Transformations and Deformations of Surfaces, Special Questions, Gosudarstv. Izdat. Tekhn. Teor. Lit., Moscow-Leningrad, 1948 (in Russian).

[32] K. Kikuchi, A family of constant mean curvature tori in $S^{3}$ with left invariant metric, in: New Approach to Differential Geometry from the Viewpoint of Integrability Theory, Tokyo Harmonic Map Club Proc. 5 (1998), R. Miyaoka and Y. Ohnita (eds.), 70-86.

[33] S. Kobayashi, Transformation Groups in Differential Geometry, Springer, 1972.

[34] M. Kokubu, On minimal surfaces in the real special linear group $S L(2, \mathbf{R})$, Tokyo J. Math. 20 (1997), 287-297.

[35] Ü. Lumiste, Normally flat submanifolds with parallel third fundamental form, Eesti NSV Tead. Akad. Toimetised Füüs.-Mat. 38 (1989), 129-138, 238. 
[36] A. Morimoto, On normal almost contact structure with a regularity, Tôhoku Math. J. 16 (1964), 90-104.

[37] H. Muto, On the stability of a three-sphere, Kōdai Math. J. 12 (1989), 109-115.

[38] K. Ogiue, On fiberings of almost contact manifolds, Kōdai Math. Sem. Rep. 17 (1965), $53-62$.

[39] B. O'Neill, The fundamental equations of a submersion, Michigan Math. J. 13 (1966), 459-469.

[40] U. Pinkall, Hopf tori in $S^{3}$, Invent. Math. 81 (1985), 379-386.

[41] T. Sakai, The contact structure on $\{S U(n+1) \times \mathbf{R} / S U(n) \times \mathbf{R}\}_{\alpha}$ and $\{S p(n) \times$ $S U(2) / S p(n-1) \times S U(2)\}_{\alpha}$ of Berger, Tôhoku Math. J. 25 (1973), 145-155.

[42] P. Sitzia, Explicit formulas for geodesics of three-dimensional homogeneous manifolds with isometry group of dimension 4 or 6, preprint, Univ. Cagliari, 1989.

[43] W. Strübing, Symmetric submanifolds of Riemannian manifolds, Math. Ann. 245 (1979), $37-44$.

[44] M. Takeuchi, Parallel submanifolds in space form, in: Manifolds and Lie Groups-Papers in Honor of Yozo Matsushima, Birkhäuser, Basel, 1981, 429-447.

[45] N. Tanaka, A Differential Geometric Study on Strongly Pseudo-Convex Manifolds, Lecture in Math. Kyoto Univ. 9, Kinokuniya Book Store, 1975.

[46] S. Tanno, Sasakian manifolds with constant $\varphi$-holomorphic sectional curvature, Tôhoku Math. J. 21 (1969), 501-507.

[47] S. Tanno, Instability of spheres with deformed Riemannian metric, Kōdai Math. J. 10 (1979), 250-257.

[48] W. M. Thurston, Three-Dimensional Geometry and Topology I, Princeton Math. Ser. 35, 1997.

[49] P. Tomter, Constant mean curvature surfaces in the Heisenberg group, in: Proc. Symp. Pure Math. 54, Amer. Math. Soc., 1993, 485-495.

[50] F. Tricerri and L. Vanhecke, Homogeneous Structures on Riemannian Manifolds, London Math. Soc. Lecture Note Series 52, Cambridge Univ. Press, 1983.

[51] H. Urakawa, On the least positive eigenvalue of the Laplacian for compact group manifolds, J. Math. Soc. Japan 31 (1979), 209-226.

[52] G. Vranceanu, Leçons de Géométrie Différentielle I, Ed. Acad. Rep. Roum., Bucarest, 1947. 